\title{
Removable of distortion by heterogeneous surface in 3-D MT inversion
}

\author{
Masanori TANI ${ }^{1}$, Hitoshi MIKADA ${ }^{1}$, Tada-nori GOTO ${ }^{1}$, Junichi TAKEKAWA ${ }^{1}$, \\ and Weerachai Siripunvaraporn ${ }^{21}$ \\ ${ }^{1}$ Dept. of Civil and Earth Res. Eng., Kyoto University \\ ${ }^{2}$ Mahidol University, Thailand
}

\begin{abstract}
In this research, we show the effects of static distortion by heterogeneous surface in 3-D MT inversion results, and propose an improved 3-D MT inversion method that can remove the effects of static distortion. We use WSINV3DMT (Siripunvaraporn et al., 2005) ${ }^{1}$ to estimate synthetic MT response and obtain inversion results. First we confirmed 3-D MT forward calculation can express static distortion on MT response. Second we applied WSINV3DMT to synthetic some MT responses that are basically same, but some false resistivity anomalies are imaged due to the distortion. As a result we conformed distinct difference between 3-D MT inversion results. We also the improved 3-D MT inversion code based on WSINV3DMT. Based on our results, we suggest that this improved inversion can effectively express the distrotion as heterogenios surface layer, and can give us more reliable resistivity structure.
\end{abstract}

\section{INTRODUCTION}

Recent years, 3-D MT inversions are widely used for geophysical investigation (e.g., Uchida and Sasaki, 2006) $)^{2}$.3-D MT inversion can get more realistic result than 2-D inversion, especially to the 3-D nature of geological structure, such as geothermal area or volcanic area(e.g. Siripunvaraporn 2005b) ${ }^{3}$. Compered with 2D MT inversions, 3-D MT inversions can recover more realistic and clear conductivity structure(e.g. Siripunvaraporn and Egbert 2000) ${ }^{4}$. However, because of the 3-D MT inversions have been applied to observed data recently, many refinements have not been considered. An effect of static distortion to a 3-D MT inversion is the one of major problems. This research reveals the effect of static distortion to 3-D MT inversion and show a refined method of a 3-D MT inversion. Static distortion is caused by local resistivity anomalies on the surface. The static distortion makes false images in the inverted result model. Originally these effects of static distortion should be reproduced as local anomaly at the surface layer model. But, because of the smoothness function, the static distortion on MT response is reproduced as gradient resistivity change at deeper area. Especially, for a resource exploration, target depth are 0 to several $\mathrm{Km}$. At those shallow depth, the effect of static distortion on inverted model is strong, enough to get false anomaly in inverted model. For those reasons, static distortion have to be removed. In order to improve 3-D MT inversion results, we refine the smoothness function to be able to produce local anomaly on the surface layer of an inverted model. In this research, we modified a 3-D MT inversion algorithm based on WSINV3DMT(Siripunvaraporn et al., 2005a) ${ }^{1}$.

\section{METHOD}

(1) Evaluation of the effect of static distortion on an inversion result model

We applied WSINV3DMT to several synthetic datasets to evaluate how local anomalies on the surface affects the result of 3-D MT inversion. The synthetic resistivity models used in this experiments are shown in Figure 1 and Figure2. Figure 1 shows resistivity structure of the under ground, which has low and high resistivity anomalies. Figure 2 shows resistivity models at the surface. Model A is without surface local heterogeneities, and model $\mathrm{B}$ is with heterogeneities. The thickness of surface blocks is $10 \mathrm{~m}$. We estimated synthetic MT response functions from these two models, model A and model B and applied WSINV3DMT to those synthetic datasets. we use WSINV3DMT to calculate synthetic data and inverse it.

(2) Removable of the effect of static distortion on an inversion result model

We applied the modified inversion method to a MT respones function calculated from model B. In the modified inversion method, static effects are expressed at the top thin layer. In our inversion algorithm, which is based on Occam's inversion (Constable et al., 1987) ${ }^{5}$, the minimization function is described as follows:

$$
\begin{array}{r}
W(\mathbf{m})=\lambda^{-1}(\mathbf{d}-\mathbf{F}[\mathbf{m}])^{T} \mathbf{C}_{\mathbf{d}}^{-1}(\mathbf{d}-\mathbf{F}[\mathbf{m}]) \\
+\left(\mathbf{m}-\mathbf{m}_{0}\right)^{T} \mathbf{C}_{\mathbf{m}}^{-1}\left(\mathbf{m}-\mathbf{m}_{0}\right)
\end{array}
$$

where $\mathbf{m}$ is the resistivity model, $\mathbf{m}_{0}$ is the prior model, $\mathbf{C}_{\mathbf{m}}$ is the model covariance matrix which defines the 


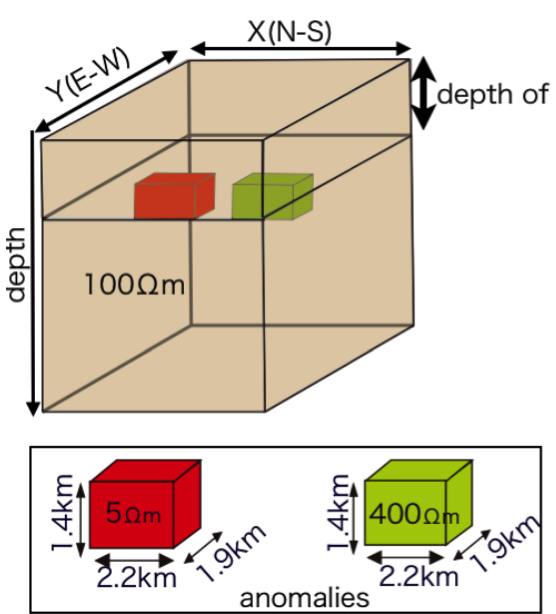

Fig. 1 Model A and B's underground resistivity structure.
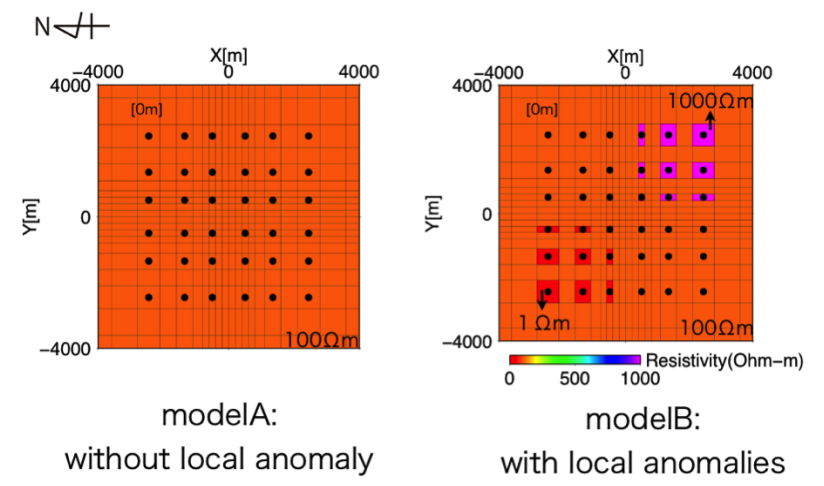

Fig. 2 Model A and B's surface resistivity structure, black dots mean observation points.

model norm, $\mathbf{d}$ is the observed data, $\mathbf{F}[\mathbf{m}]$ is the model response, $\mathbf{C}_{\mathbf{d}}$ is the data covariance matrix, and $\lambda^{-1}$ is a Lagrange multiplier. The model covariance matrix characterizes the smoothness of resistive variation relative to the base model. In order to express static effects at surface layer in the inversion procedure models, we changed the model covariance matrix. In the new covariance matrix, the weighting of the smoothness between the first layer and second layer becomes smaller (10\%) than original one. Fig.3 is simple image of our method.

We apply our method to another synthetic MT response. In this examination, the synthetic model witch be used to estimate synthetic observed MT response, is $100 \Omega m$ homogenous structure. First, we calculate MT response from the model C, shown in Fig.4. Second we calculate synthetic distorted MT response, as follows. Finally we apply 3-D MT inversions(original WSINV3DMT and Our method).

$$
\mathbf{Z}=\left(\begin{array}{cc}
Z_{x x} & Z_{x y} \\
Z_{y z} & Z_{y y}
\end{array}\right)
$$

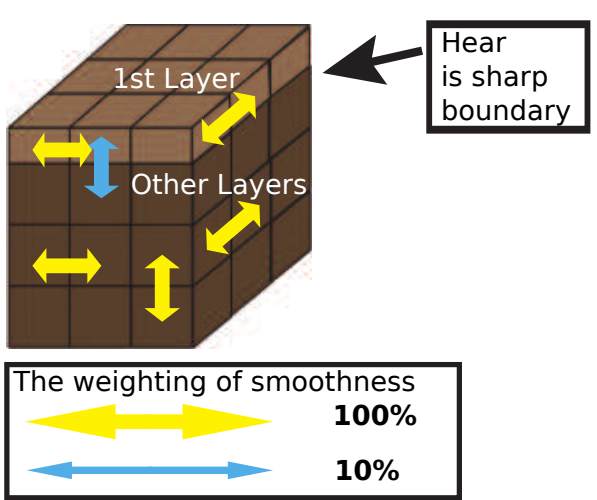

Fig. 3 The explanation of our new method.

$$
\mathbf{C}=g \cdot \mathbf{T} \cdot \mathbf{S} \cdot \mathbf{A}
$$

with this matrix $\mathbf{C}$ we calculate

$$
\mathbf{Z}^{\text {obs }}=\mathbf{C Z}
$$

then synthetic observation MT response $\mathbf{Z}^{\text {obs }}$ are omitted.

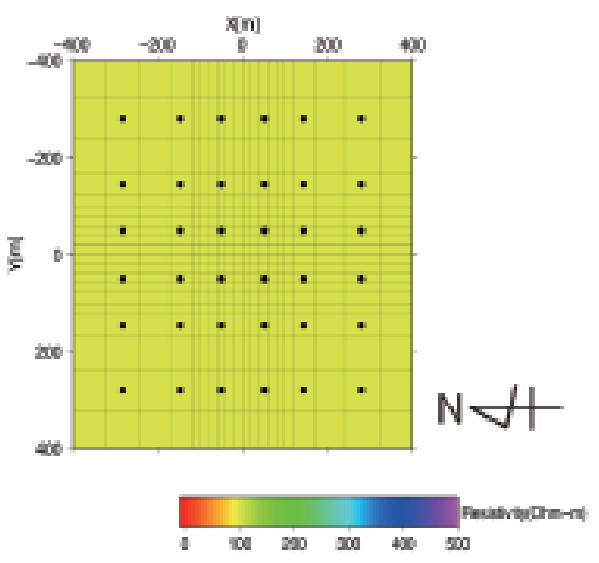

Fig. 4 Model C. Black dots mean observation points.

In this study, we determine $\mathbf{C}$ components by results of forward calculation as shown Fig.5.

\section{RESULTS AND DISCUSSIONS}

\section{(1)The effect of static distortion on an inversion result model.}

As shown in Fig.7, the model of datasets calculated from MT responses on the model B has false anomalies. This result shows inverted model from MT response with static distortion makes false anomalies at various depth levels and this inverted models show unreasonable resistivity structure compered with the result of model A, without a local surface anomaly (Fig.6).

Fig.?? is the inversion result from MT response with synthetic distortion matrix. This result also show the effect of static distortion clearly. Inversion algorithm fail to express local anomaly, and make false anomaly 
a)

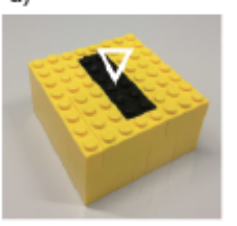

b)

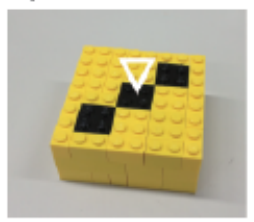

a)

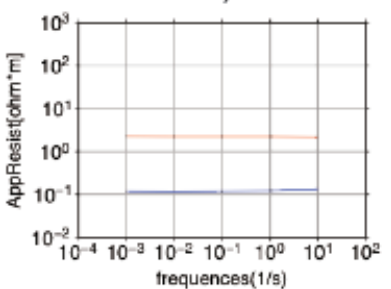

PHASE

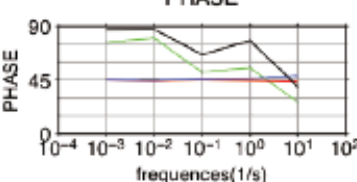

b)

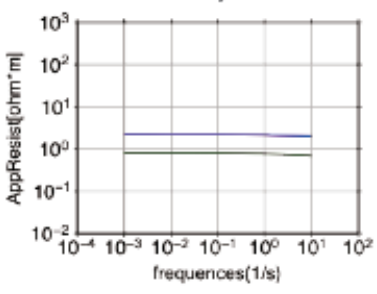

PHASE
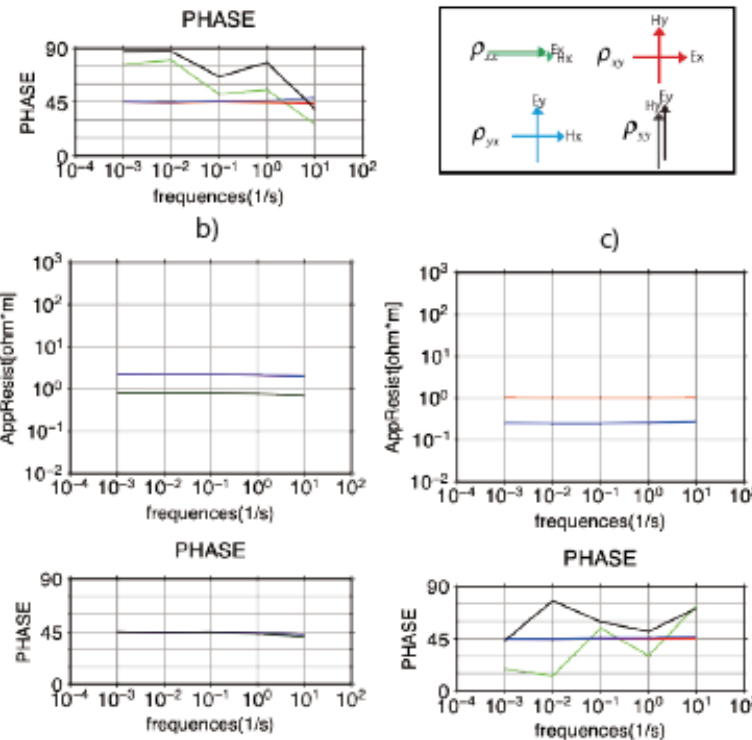

c)

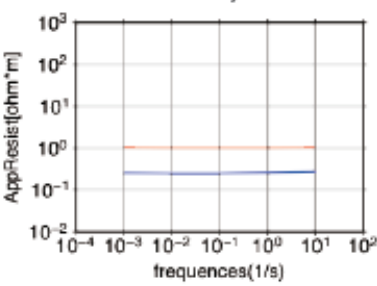

PHASE

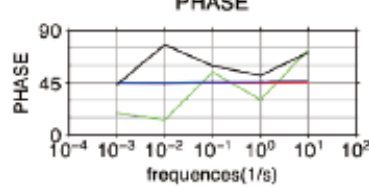

Fig. 5 Exsamples of MT response calculated from homogeneous model with local anomaly on the surface.

at deeper area.

\section{(2) Removable of the effect of static distortion on an inversion result model}

Fig.9 shows the inverted model of MT response function calculated from model B with the new model covariance. In this result, the inverted resistivity structure model becomes more realistic compared with the model shown in Fig.7 which is the inverted model from same datasets and with the ordinal model covariance - As shown in Fig.7 static distortion caused by local surface anomalies recovered at the top layer and false anomalies at deeper area are removed. Fig.10 is inversion result from MT response with synthetic distortion matrix. This result also shows better resistivity model result than inversion result with original WSINV3DMT(Fig.??). Although our modified inversion indicates the effectiveness to the static distortion, further discussion should be required. For example, we simply reduce the weight of the top layer in the smoothness calculation with $10 \%$ of original one. More suitable weighting should be discussed for avoiding too

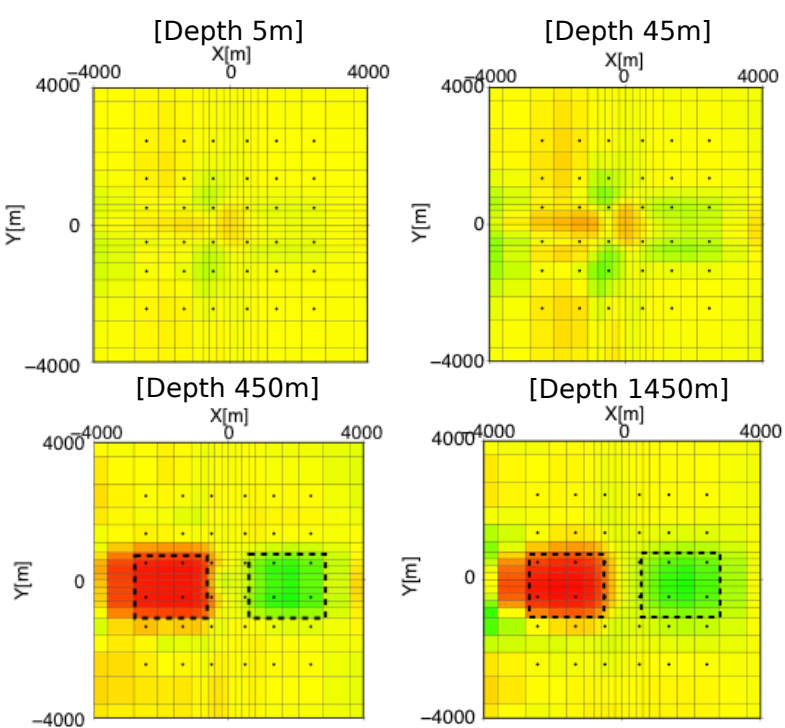

Fig. 6 Conventional inversion result with out inversion method, of model A's MT response function.
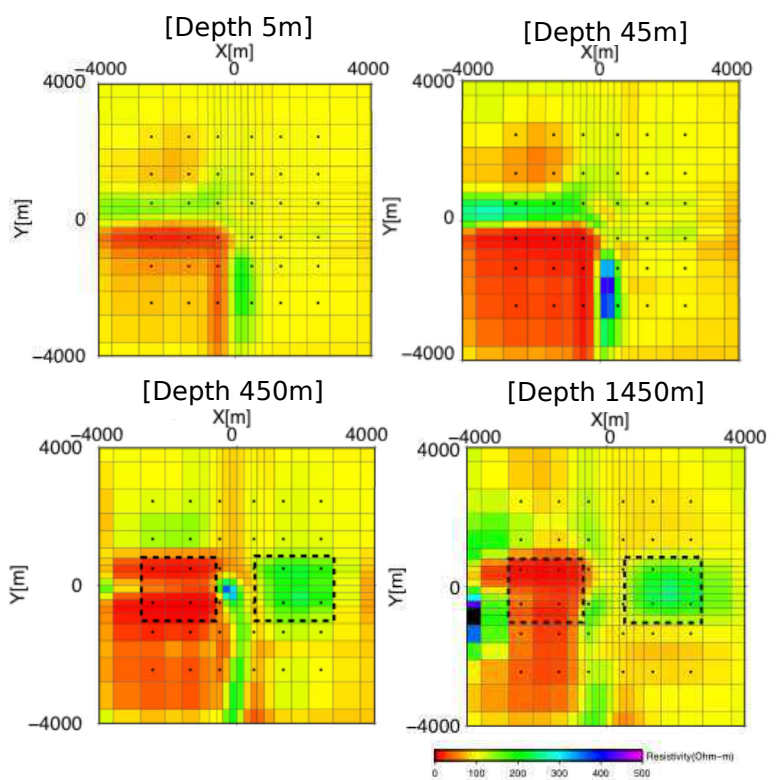

Fig. 7 Conventional inversion result of model B's MT response function with original WSINV3DMT.

complex resistivity structure near the surface.

\section{CONCLUSION}

In this paper, We revealed that that static effects make false resistivity anomalies in inverted model. As shown in Fig.7 and 8, static distortion on MT response function effects deep area of inversion result because of smoothness function. This effect are reproduced as false anomalies, which are too big to be ignored. This 


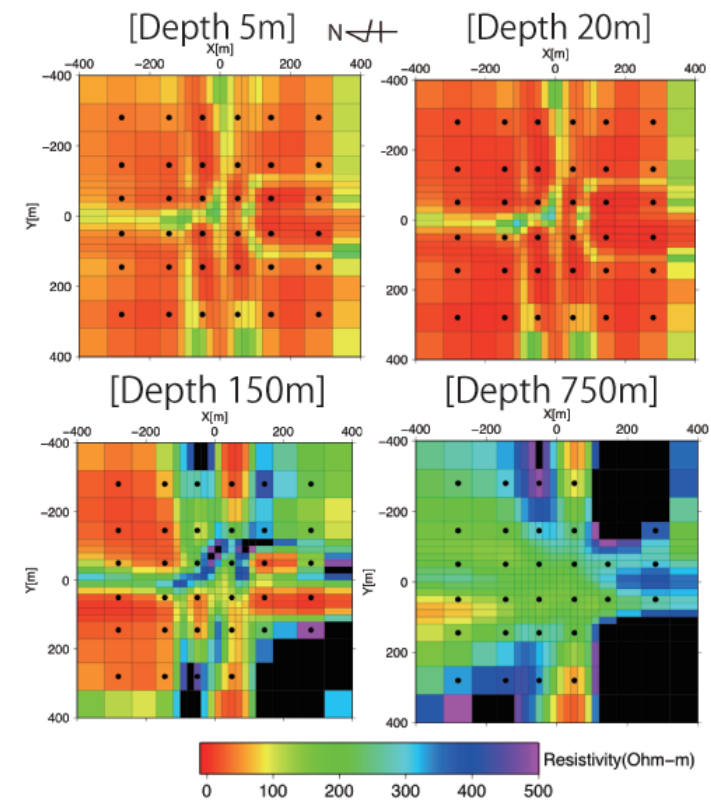

Fig. 8 Conventional inversion result, with out inversion method, of model C's MT response function which distorted by synthetic distortion matrix.

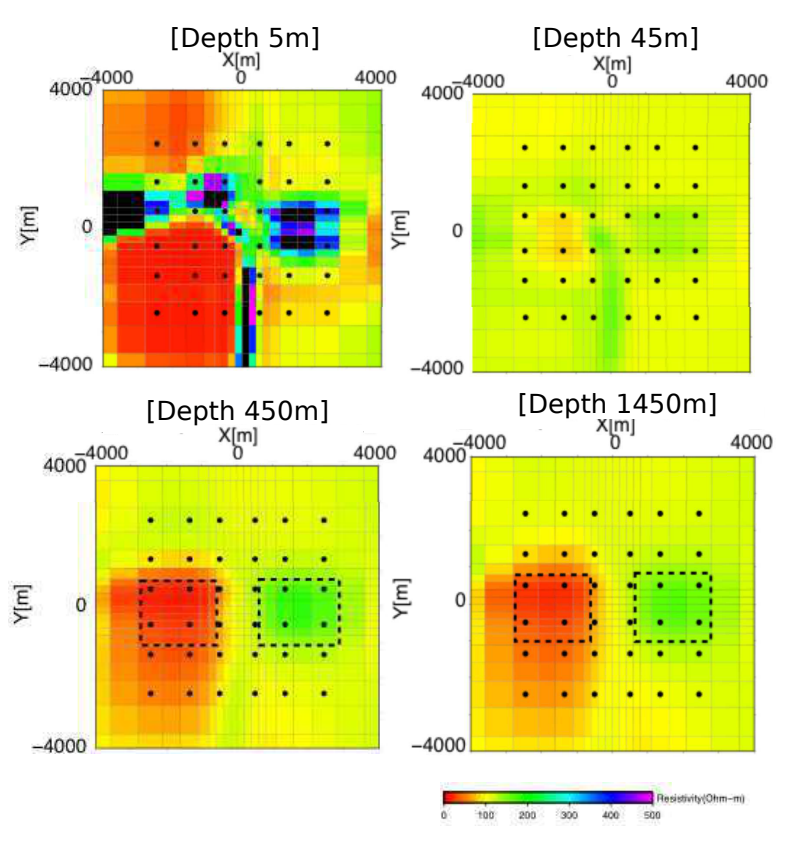

Fig. 9 Our new inversion result of model B's MT response function with our inversion method.

problem should not be ignored to know realistic resistivity structure of the underground. The result of modi- fied inversion method is more reliable than the result of original inversion method. This result means the new method is useful to deal with static distortion. Since the thin surface layer can express the static distortion, our method will also reduce the distortion effects in 3D MT inversion.

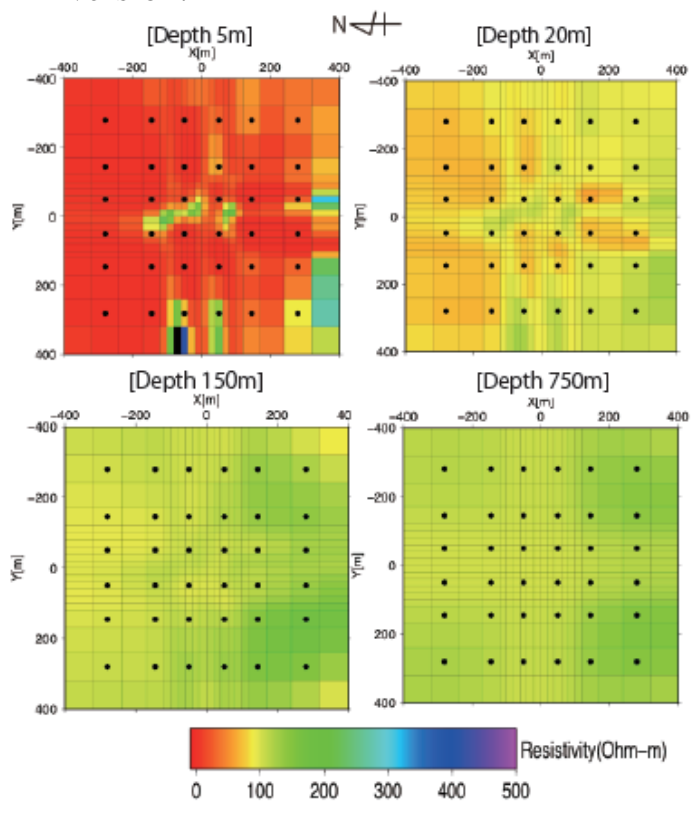

Fig. 10 Our new inversion result, with out inversion method, of model C's MT response function which distorted by synthetic distortion matrix.

\section{REFERENCES}

${ }^{1}$ Siripunvaraporn. W, et al. (2005a): ThreeDimensional Magnetotelluric inversion: Data Space Method, Physics of the Earth and Planetary Interiors, 150, 3-14.

${ }^{2}$ Uchida, T., and Sasaki, Y., (2006): Stable 3D inversion of MTdata and its application to geothermal exploration, Exploration GeoPhysics, 37, 223-230.

${ }^{3}$ Siripunvaraporn. W, et al, (2005b): Interpretation of two-dimensional magnetotelluric profile data with three-dimensional inversion: synthetic examples, Geophys. J. Int.,

${ }^{4}$ Siripunvaraporn. W. and G. Egbert, (2000): An efficient data-subspace inversion method for 2-D magnetotelluric data, Geophysics, 65, 791-803.

${ }^{5}$ Constable, S., et al, (1987): Occams' inversion: A practical algorithm for generating smooth models from electromagnetic sounding data, GEOPHYSICS. 52, 289-300. 160, 804-814. 